\title{
Carcinoma Cervix in Pregnancy Presenting as Antepartum Haemorrhage: A Case Report
}

\author{
Authors \\ Sayantan Sen, Arijit Dutta, Vasundhara Goswami, Sumit Kumar Das \\ Burdwan Medical College, WBUHS \\ Email: sayantan.sen88@gmail.com
}

\begin{abstract}
Cervical Cancer is the most common genital malignancy associated with pregnancy. It usually presents in the 1st trimester and at an early stage. Presentation can be variable ranging from pain abdomen to bleeding per vaginum. Management protocols are based on the gestational age at presentation and stage of the disease. A 28 year old, P 2+0,3rd Gravida at 30 weeks of gestation presented with bleeding per vaginum and pain lower abdomens for 4 weeks. She was diagnosed as a case of stage IIB cervical carcinoma. After counseling the patient regarding prognosis, she was planned for caesarean section and given two doses of Inj. Betamethasone (12mg).A classical section was performed and she delivered a live girl weighing $1.5 \mathrm{~kg}$ who was sent to the sick newborn care unit (SNCU) after initial resuscitation. She developed bilateral inguinal lymphadenopathy and unilateral oedema of thigh and foot on the 10th post-operative day. Magnetic Resonance Imaging was done and suggested deep vein thrombosis. Attending physician advised anticoagulant therapy and heparin injection was started immediately. Her newborn was released from the SNCU 20 days after delivery and breastfed well. She was discharged the next day and advised to attend the radiotherapy outpatient department. She was advised radiotherapy after involution of uterus.
\end{abstract}

\section{Introduction}

Cervical cancer is the most common malignancy in pregnancy with an estimated incidence of 0.8 to 1.5 cases per 10000 births $^{[1]}$. The incidence of carcinoma cervix in India is high due to poor socioeconomic condition and early age of marriage. It is the commonest cancer cause of death among women in developing countries and 122,844 women are diagnosed with cervical cancer and about 67,477 die from the disease in India every year $^{[2,3]}$.

Cancer screening protocols have reduced its incidence over the last couple of decades.
In pregnancy, it presents usually in early stage. This is probably due to early screening or difficulty of conception in advanced stage. Common symptoms include bleeding per vaginum, lower abdominal pain or foul smelling vaginal discharge.

Diagnosis is by PAP smear and Colposcopy in case of abnormal smear. Endocervical curettage is contraindicated in pregnancy. Diagnostic conization can be performed if limits of the lesion are not visible but should be avoided in the 1st trimester. Diagnosis may be delayed due to confusion with other early pregnancy complications. 
Treatment modality depends on the stage of disease, the gestational age at presentation and the patients' desire. Advanced stage warrants immediate termination followed by either radical hysterectomy or primary radiotherapy depending on disease stage. A more conservative approach can be followed in early disease. Follow up of the disease is essential and adjuvant therapy with radiation has become the standard after uterine involution.

\section{Case Report}

A 28 year old 3rd Gravida at 30 weeks of gestation presented with bleeding per vaginum and pain lower abdomen for 4 weeks. She was referred from a primary health centre as a case of antepartum haemorrhage. She belonged to a tribal community. First sexual intercourse was at 20 years of age. There was no family history of malignancy. General examination revealed a cachectic woman with moderate pallor. Radial pulse rate was 90 beats per minute and blood pressure $110 / 76 \mathrm{~mm}$ of $\mathrm{Hg}$. Fundal height corresponded to 30 weeks of gestation and fetal heart rate was 130/min and regular. On per speculum examination, a fungating friable growth was seen arising from the cervix which bled on touch and was associated with foul smelling discharge (as shown in Fig. 1)

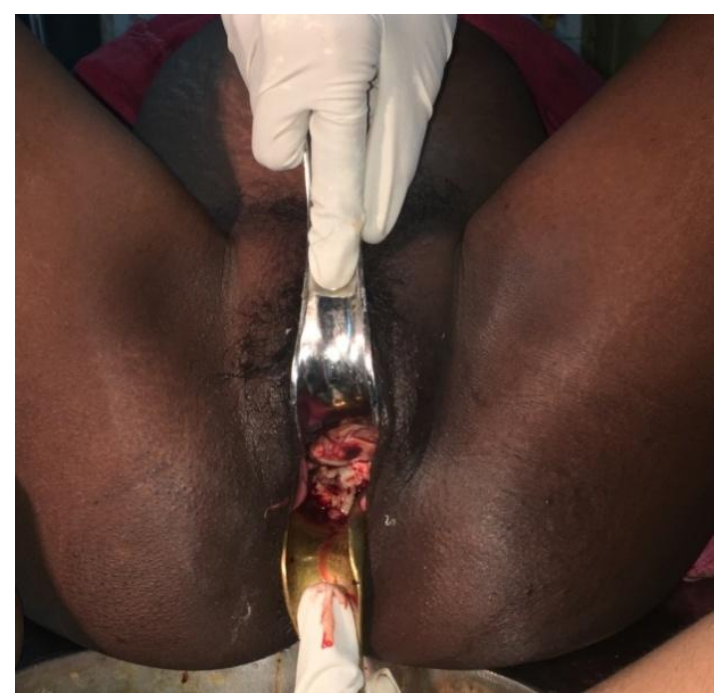

Figure 1: Per speculum examination showing a fungating growth from the cervix
The mass did not extend to the vagina and no lymph node could be palpated. On per vaginum and rectal examination parametrium was involved and clinical staging was IIB. Histopathology of the biopsy sample showed Squamous Cell Carcinoma (large cell) with extensive necrosis.

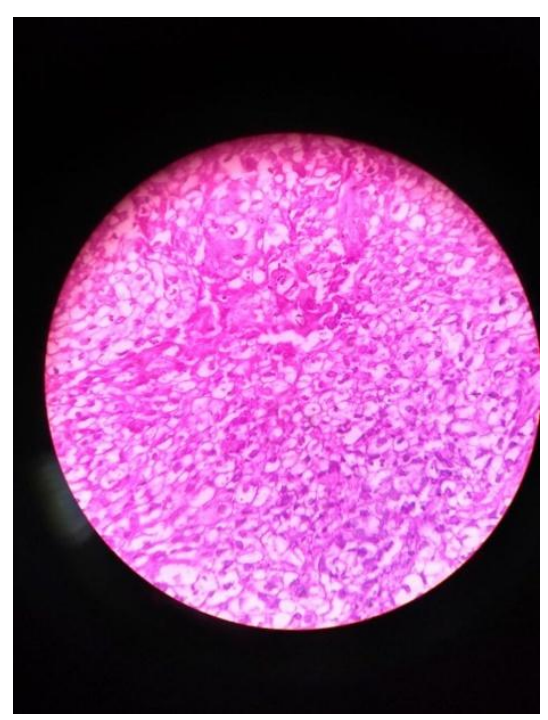

Figure 2: Histopathology of Biopsy Sample using Haematoxylin and Eosin stain showing Squamous Cell Carcinoma

Obstetric ultrasound showed a single live fetus at 29 weeks 5 days gestation in breech position with adequate liquor and fundal placenta. After counselling the relatives regarding prognosis of mother and baby, she was planned for caesarean section and given two doses of Inj. Betamethasone (12mg).On section, uterus and adnexa were normal and a cervical mass could be palpated through the Pouch of Douglas. No enlarged lymph nodes were palpable. She delivered a live girl, $1.5 \mathrm{~kg}$, through a classical incision. APGAR score was 8.She was sent to the SNCU, after initial resuscitation, for further management. Immediate post operative period was uneventful. Stitches were removed on the 8 th post-operative day.

She developed bilateral inguinal lymphadenopathy and unilateral edema of thigh and foot on the 10th post operative day .Contrast enhanced CT scan and doppler study was done and suggested deep vein thrombosis in multiple veins. Attending physician advised anticoagulant therapy and hepa- 


\section{JMSCR Vol||06||Issue||06||Page 447-450||June}

rin injection was started immediately. Coagulation profile was normal.

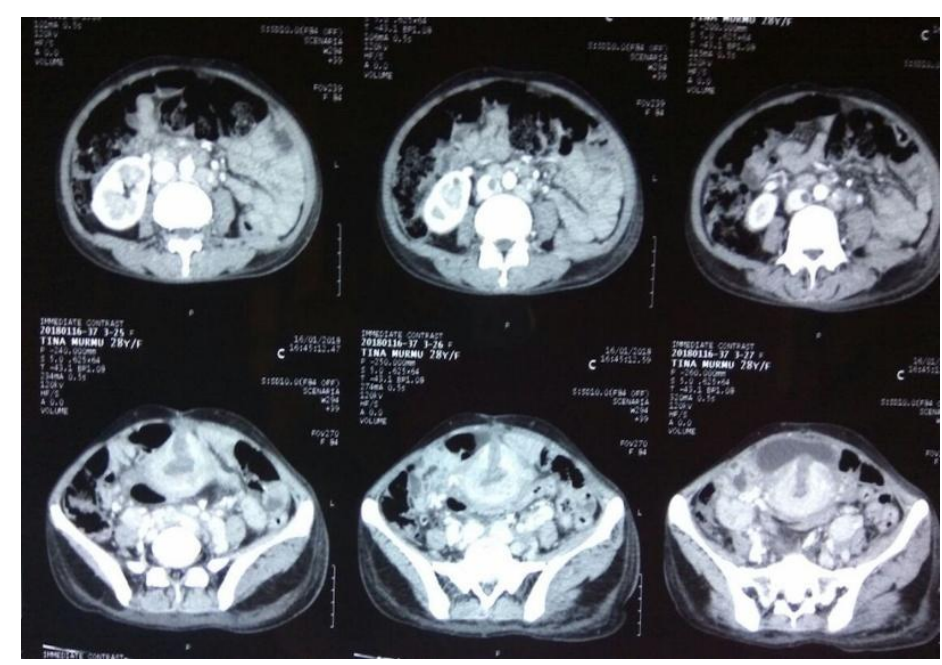

Figure 3 : Contrast enhanced CT scan

Her newborn was released from the SNCU 20 days after delivery and breastfed well.

She was discharged the next day and advised to attend the radiotherapy outdoor. She was advised radiotherapy after involution of uterus.

\section{Discussion}

Invasive cervical cancer during pregnancy poses a dilemma to the physician. On one hand is the prognosis of the fetus and on the other, the survival of the mother and the increased risk of complications due to delay in treatment initiation. The clinical course and prognosis of the disease are not affected by pregnancy ${ }^{[4,5]}$. However, the presentation may be delayed and so can the treatment initiation. The average duration of symptoms before diagnosis of cervical cancer in pregnancy is 4 and half months.

Germann et $\mathrm{al}^{[6]}$ describe a series of 21 cases of cervical cancer managed during pregnancy or the postpartum period. They point out that, despite numerous publications, questions remain regarding cervical cancer in pregnancy.

Treatment should be individualized and based on the stage of cancer, the woman's desire to continue pregnancy, and the risks of modifying or delaying therapy during pregnancy. Early cancer cervix in pregnancy can be managed conservatively by continuing the pregnancy till fetal lung maturity, delivering by elective classical section, followed by radical hysterectomy and adjuvant chemoradiation. Management of advanced disease can be a challenging task. Before fetal viability, the best option is primary chemoradiation following which abortion usually follows. After fetal viability, elective classical caesarean section can be done without delay and post op radiation given after involution.

In our patient, presentation was quite late as she did not have regular antenatal checkups and was ignorant about the symptoms. She presented at a stage where surgical management was not possible and palliative radiation was the only option. Even though we delivered the baby at 30 weeks, she survived without any serious complications thanks to the Department of paediatrics.

Deep vein thrombosis is a known complication of cervical carcinoma. The development of DVT in conjunction with a gynecologic malignancy connotes a poor prognosis, especially in patients with cervical cancer. It is possible that this poor prognosis is related to the pathophysiology that results in venous thrombosis and not just the presence of cancer $^{[7]}$. It was effectively managed by anticoagulation and she was asymptomatic on follow up.

\section{Conclusion}

The management of carcinoma cervix in pregnancy is still a grey area due to the lack of any standardized treatment protocol. This is more so in patients presenting at an advanced stage. Whether delaying treatment to save the fetus is an acceptable option, is still a matter of debate. The treatment is thus individualized taking into account the patients' desire as well as the available facilities.

\section{References}

1. Duggan B, Muderspach LI, Roman LD, et al. Cervical can- cer in pregnancy: reporting on planned delay in therapy. Obstet Gynecol 1993;82:598-601.

2. Denny L. Cervical cancer: prevention and treatment. Discov Med. 2012;14:125-131. 
JMSCR Vol||06||Issue||06||Page 447-450||June

3. ICO Information Centre on HPV and cancer (Summary Report 2017-07-27).Human Papillomavirus and Related Diseases in India. 2017.

4. Catarino R, Petignat $\mathrm{P}$, Dongui G, Vassilakos P. Cervical cancer screening in developing countries at a crossroad: Emerging technologies and policy choices. World J Clin Oncol 2015;6: 281-90.

5. Smith L, Danielsen B, Allen M, Cress R. Cancer associated with obstetric delivery: results of linkage with the California cancer registry. Am J obstet Gynecol 2003; 189:1128-1135.

6. Germann N, Haie-Meder C, Morice P et al. Management and clinical outcomes of pregnant patients with invasive cervical cancer. Ann Oncol 2005;16:397-402.

7. Morgan MA, Iyengar TD, Napiorkowski BE, Rubin SC, Mikuta JJ.The clinical course of deep vein thrombosis in patients with gynecologic cancer.Gynecol Oncol. 2002;84:67-71. 